\title{
Do we need more tools in the management of bronchiolitis or are only oxygen, salt water and a cool head required?
}

Acute bronchiolitis is a common cause of lower respiratory tract infection and is associated with considerable morbidity. There is an increase in the number of hospitalisations and, because of the improved quality of neonatal care, a number of neonatal intensive care unit (ICU) graduates present with this illness. Prophylactic therapy with palivizumab is not curative and its use is extremely limited in low- to middle-income countries, resulting in a number of children presenting with bronchiolitis, particularly in the respiratory syncytial virus season. A subset of these children may develop severe disease without identified risk factors. As the majority are seen at a primary healthcare level, there is a need to find tools that can assist in the prediction of disease severity.

Capnography is used in the assessment of percutaneous end-tidal $\mathrm{CO}_{2}\left(\mathrm{ETCO}_{2}\right)$ measurements and is useful for the measurement of ventilation, perfusion and acid base status. Jacob et al. ${ }^{[1]}$ conducted a prospective, single-blind cohort study in children younger than 2 years presenting to the emergency department (ED) with bronchiolitis. Their primary outcome was the correlation between the $\mathrm{ETCO}_{2}$ and the clinical decision of hospital admission and discharge. Their secondary outcome measure was the correlation of $\mathrm{ETCO}_{2}$ upon arrival at the ED and clinical measures of bronchiolitis severity. They enrolled 114 children over a 1-year period. Physicians who made decisions on admission or discharge were blinded to the $\mathrm{ETCO}_{2}$ results. $\mathrm{ETCO}_{2}$ measurements were also repeated prior to discharge.

The mean age of the study population was 6.9 months; $16 \%$ were ex-premature, with $21 \%$ having had a previous episode of wheezing. The median $\mathrm{ETCO}_{2}$ upon arrival at the $\mathrm{ED}$ was 34 (range 24 - 65) mmHg. $\mathrm{ETCO}_{2}$ values upon admission or discharge were not statistically different among patients hospitalised and those discharged from the ED. There was also no correlation between capnometry readings on admission and the number of oxygen desaturation days, nor with the length of hospitalisation. The Wang clinical respiratory severity score was found to predict the need for nasogastric tubes, oxygen desaturation days and length of hospitalisation.

The authors concluded that $\mathrm{ETCO}_{2}$ was not useful for predicting the need for hospital admission or discharge. The clinical severity score was the most consistent predictor of significant outcome. Again, the message for the management of acute bronchiolitis is probably that the best tool is what lies between the physician's ears, and not technology.

\section{R Masekela}

Paediatric Pulmonologist and Head of Department of Maternal and Child Health, Nelson R Mandela School of Clinical Medicine, University of KwaZuluNatal, Durban, South Africa 1. Jacob R, Bentur L, Brik R, Shavit I, Hakim F. Is capnometry helpful in children with
bronchiolitis? Respir Med 2016;113:37-41. DOI:10.1016/j.rmed.2016.02.007

S Afr Respir J 2016;22(2):47. DOI:10.7196/SARJ.2016.v22i2.84 